\title{
Exploring the Awareness and Acceptability of Screening Methods for Anal Intraepithelial Neoplasia in the HIV-positive Men who have Sex with Men Population
}

\author{
Maximilian Johnson 1., Dr. Larissa Mulka 1. 2., Dr. Daniel Richardson 1. 2. \\ 1. Brighton \& Sussex Medical School, Brighton \\ 2. Brighton \& Sussex University NHS Trust, Brighton
}

\section{Anal Intraepithelial Neoplasia: A Rising Concern?}

Rates of AIN are increasing globally in parallel with anal SCC diagnoses, which have risen by 56\% in the UK since 1990.

There are multiple risk factors but three are most significant:

1. High risk sexual behaviours

2. Exposure to HPV, particularly strains 16 and 18

3. HIV infection

HIV positive men-who-have-sex-with-men (MSM) are at a $\mathbf{> 1 0 0}$-fold increased risk of developing anal SCC compared to male controls.

No formal screening programme exists in high risk groups due to a lack of evidence for their efficacy.

\section{Methods}

A targeted patient information leaflet was designed and distributed to HIV-positive MSM prior to routine clinic appointments. Having read this, respondents were invited to complete a survey assessing existing knowledge and the utility of the PIL.

\section{Results}

172 surveys were completed with an acceptance rate of $87.0 \%$ and a modal age range of $45-54(n=50)$.

\section{Awareness}

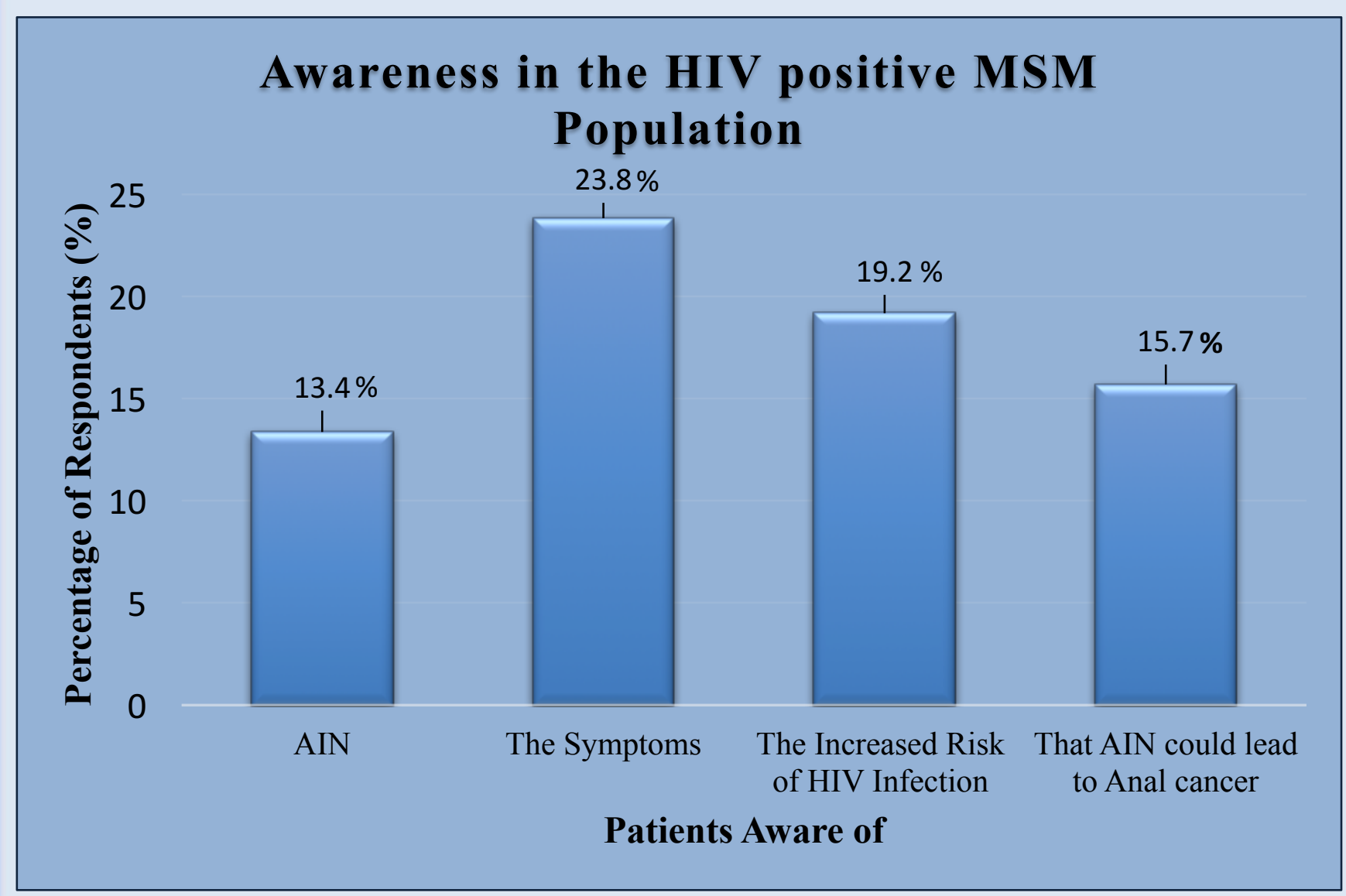

Awareness of AIN was very low:

- Only $13.4 \%$ of respondents had heard of AIN before

- Interestingly more knew about: the symptoms (23.8\%), the increased risk that HIV infection posed (19.2\%) and that AIN could lead to anal SCC (15.7\%)

- Only $23(13.4 \%)$ respondents self-examined regularly, 55 (32.0\%) did so rarely and 94 (54.6\%) never did so

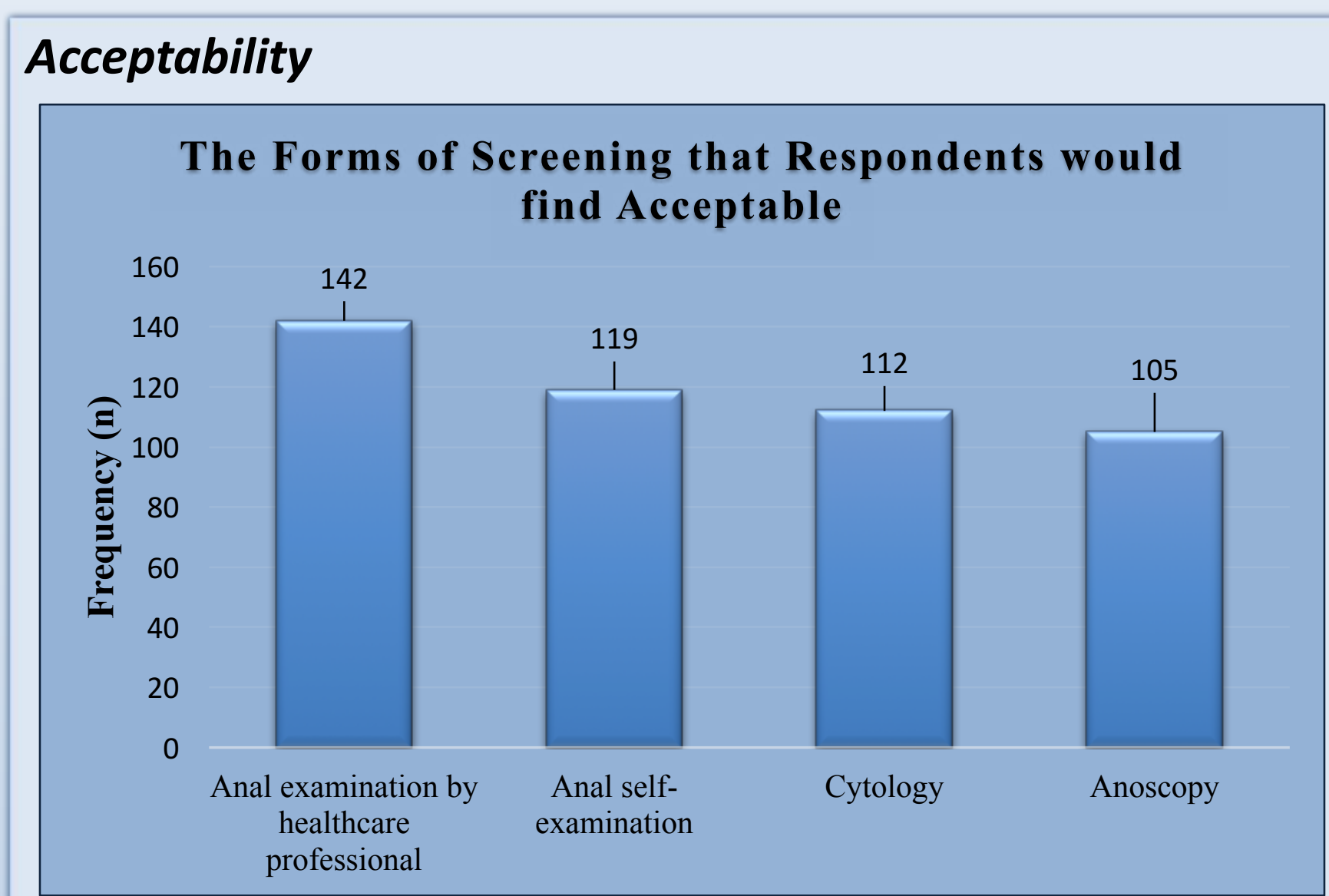

Support for a screening programme was high:

- $83.1 \%$ of respondents stated they would participate

- The preferred method was anal examination by a healthcare worker $(82.6 \%)$

\section{The Patient Information Leaflet}

$84.9 \%$ respondents read the leaflet:

All found it was well laid out, easy to read and understand $14.4 \%$ said that after reading it they did not feel confident performing anal self-examination

\section{Conclusions}

The short study period limited the scope and sample size but the findings serve to inform the development and implementation of a screening service for AIN.

Healthcare workers have a duty to promote self-examination and, should evidence emerge for it's efficacy, they should offer anal examinations at patients' routine appointments.

Knowledge of AIN is low in this high-risk group and there is a clear need for education. This could be achieved using a patient information leaflet such as the one utilised in this study.

Support for a screening programme is strong and these findings predict good patient uptake should a screening programme be introduced.

Current Knowledge2

HIV positive MSM have a higher risk of developing AIN and anal SCC

There is no evidence for the efficacy of screening methods for AIN

HPV infection by high-risk strains is the main factor in the development of AIN. There is currently a vaccine for this but it is not offered to men despite BASHH recommendations

\section{Knowledge gained}

Knowledge of AIN is low in HIV positive MSM and there is a clear need for education Anal examination by Healthcare Workers (HCWs) would be the most acceptable form of screening Once educated in how to perform anal self-examination many patients would be more prepared to, although some would still prefer to be examined by HCWs 